\title{
Mycoplasma Pneumoniae and Antibodies against Galactocerebroside in a 9-Year-Old Boy with Encephalitis
}

\author{
Joost Smolders ${ }^{1}$ Bart C. Jacobs ${ }^{2}$ Anne P. Tio-Gillen ${ }^{2}$ Frouke Nijhuis $^{3}$ Aad Verrips ${ }^{1}$ \\ ${ }^{1}$ Department of Pediatric Neurology, Canisius Wilhelmina Hospital, \\ Address for correspondence Aad Verrips, MD, PhD, Department of \\ Nijmegen, the Netherlands \\ 2 Departments of Neurology and Immunology, Erasmus MC, \\ University Medical Center Rotterdam, the Netherlands \\ ${ }^{3}$ Department of Clinical Neurophysiology, Canisius Wilhelmina \\ Pediatric Neurology, Canisius Wilhelmina Ziekenhuis, Weg door \\ Jonkerbos 100, 6532 SZ Nijmegen, the Netherlands \\ (e-mail: a.verrips@cwz.nl).
}

Neuropediatrics 2019;50:54-56.

\begin{abstract}
Keywords

- encephalitis

- mycoplasma pneumoniae

- galactocerebroside

We report the case of a 9 year-old boy, presenting with an acute encephalitis with cerebrospinal fluid pleiocytosis. MRI showed T2/FLAIR (fluid attenuated inversion recovery) hyperintense signals of basal ganglia and cortex, EEG (electro encephalogram) showed diffuse slowing with epileptic discharges. A repetitively elevated $\operatorname{lgM} /$ $\lg G$ serologic response against Mycoplasma pneumoniae was observed with polymerase chain reaction in serum and cerebrospinal fluid remaining negative. No other pathogen or antigen could be identified. High IgG and IgM levels against the glycolipid galactocerebroside were detected in serum but not in CSF. After treatment with corticosteroids, the patient fully recovered. Brain MRI and EEG investigation returned completely normal. Besides a primary infection of the central nervous system, $M$. pneumoniae is associated with a parainfectious encephalitis in children which may be mediated by antibodies to galactocerebroside.
\end{abstract}

A 9-year-old boy presented at our emergency department with complaints of fever, vomiting, and generalized weakness since 2 days. His mother witnessed an episode with twitches of the mouth, right arm and right leg with deviation of the eyes to the right, lasting for less than a minute, followed by confusional behavior. A similar event was seen at our emergency department. The boy had no relevant medical history, no previous seizures during fever, and used no medications. Parents were of mixed Caucasian-far east Asian descent, and also had no relevant medical history. At neurological examination, the child was cardiopulmonary stable with a temperature of $38.6^{\circ} \mathrm{C}$. After waking him from sleep, he was well orientated and alert, without meningeal signs. Further neurological examination revealed no abnormal signs. Symptoms and physical examination were not suggestive of a pneumonia. A third seizure was observed

received

July 25, 2018

accepted after revision

October 15, 2018

published online

November 26, 2018

after the examination. Laboratory tests revealed a leukocytosis of $17.0 \times 10^{7} / \mathrm{L}$ (predominantly elevated neutrophils and monocytes) and elevated $C$ reactive protein of $23 \mathrm{mg} / \mathrm{L}$ with a normal blood glucose level of $5.9 \mathrm{mM}$. Cerebrospinal fluid (CSF) examination showed $166 \times 10^{6} / \mathrm{L}$ leukocytes, elevated lactate of $2.3 \mathrm{mM}$ with normal total protein and glucose levels ( $0.3 \mathrm{~g} / \mathrm{L}$ and $2.4 \mathrm{mM}$, respectively). Microscopic examination of CSF revealed no bacteriae for Gram staining. With an initial differential diagnosis of epileptic seizures as a possible sign of a bacterial meningitis/encephalitis, the patient was treated with ceftriaxone, dexamethasone and levetiracetam, and subsequently admitted to our pediatric ward. Observation during the next 3 days revealed transgressive behavior: in contrast to known personality by parents, the patient uttered frequently very rude language/cursed, was obstinate and tired, with a remarkably

(c) 2019 Georg Thieme Verlag KG Stuttgart · New York
DOI https://doi.org/ 10.1055/s-0038-1676054. ISSN 0174-304X. 


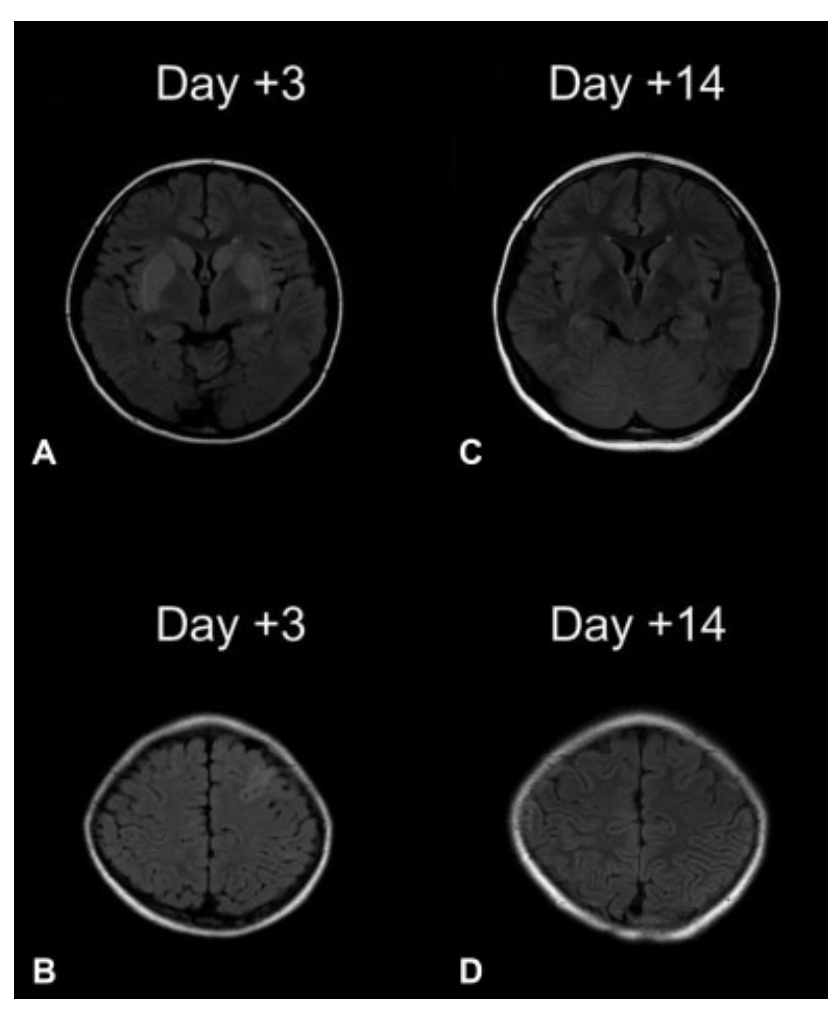

Fig. 1 FLAIR-weighted MRI-image of our patient 3 days (A, B) and 14 days $(C, D)$ after presentation. Please not the hyperintense aspect of bilateral basal ganglia (A) and left-frontal cortex (B) at presentation, with complete normalization during follow-up (C, D). FLAIR, fluid attenuated inversion recovery; MRI, magnetic resonance imaging.

large appetite. Initially, motor seizures with deviation of the eyes to the right were observed. EEG (electro encephalogram) examinations revealed a slow background rhythm of 7-8 $\mathrm{Hz}$ with prominent delta activity in the frontocentral regions ( - Fig. S1A; supplementary figure is available only in the online version) and a paroxysmal build-up of rhythmic epileptic activity (-Fig. S2: supplementary figure is available only in the online version).

CSF cultures for bacteriae remained negative and CSF polymerase chain reaction (PCR) for Herpes Simplex virus type 1 and 2, Varicella Zoster Virus, and enterovirus were negative. A brain magnetic resonance imaging (MRI) scan showed a remarkable T2 and FLAIR (fluid attenuated inversion recovery) hyperintense signal of basal ganglia and focal cortical regions (- Fig. 1A and B). Paraneoplastic antineuronal and autoantibodies were absent in blood and CSF, including anti-contactin-associated protein-like 2 (CASPR2), anti-leucine-rich glioma inactivated-1 (LGI1), anti-Nmethyl-d-aspartate receptor (NMDAR) and, anti-myelin oligodendrocyte glycoprotein (MOG). Serologic examination revealed no current infection with Rickettsia, Borrelia burgdorferi, Tick-borne encephalitis, Cytomegalovirus or EpsteinBarr Virus. An agglutination assay showed a very high serum IgM/IgG response against Mycoplasma pneumoniae (titer 1280, 3 days after presentation; titer 640, 18 days after presentation; cut-off for positive $\geq 80$ ). M. pneumoniae PCR in serum and CSF was negative, a PCR on respiratory samples was not performed. We tested the presence of antigalactocerebroside (anti-GalC) antibodies in the serum of our patient and found a marked elevation of both IgG and IgM levels ( +7.5 and +11.7 SD [standard deviation] above mean, respectively; - Fig. 2A). Only a small amount of CSF was available for the GalC assays, no clear elevation of antiGalC IgG or IgM was found (+ 2.1 and -0.2 SD from mean, respectively, -Fig. 2B). With the working diagnosis $M$. pneumoniae-associated parainfectious encephalitis, the patient was treated with methylprednisolone $20 \mathrm{mg} / \mathrm{kg} / \mathrm{day}$ for 3 days. Complaints resolved quickly, no seizures were observed, and our patient could leave our ward 10 days after presentation at the emergency department. A repeated brain MRI scan 14 days after presentation was completely normal ( - Fig. 1C and D), as well as the EEG registration (- Fig. S1B; supplementary figure is available only in the online version).
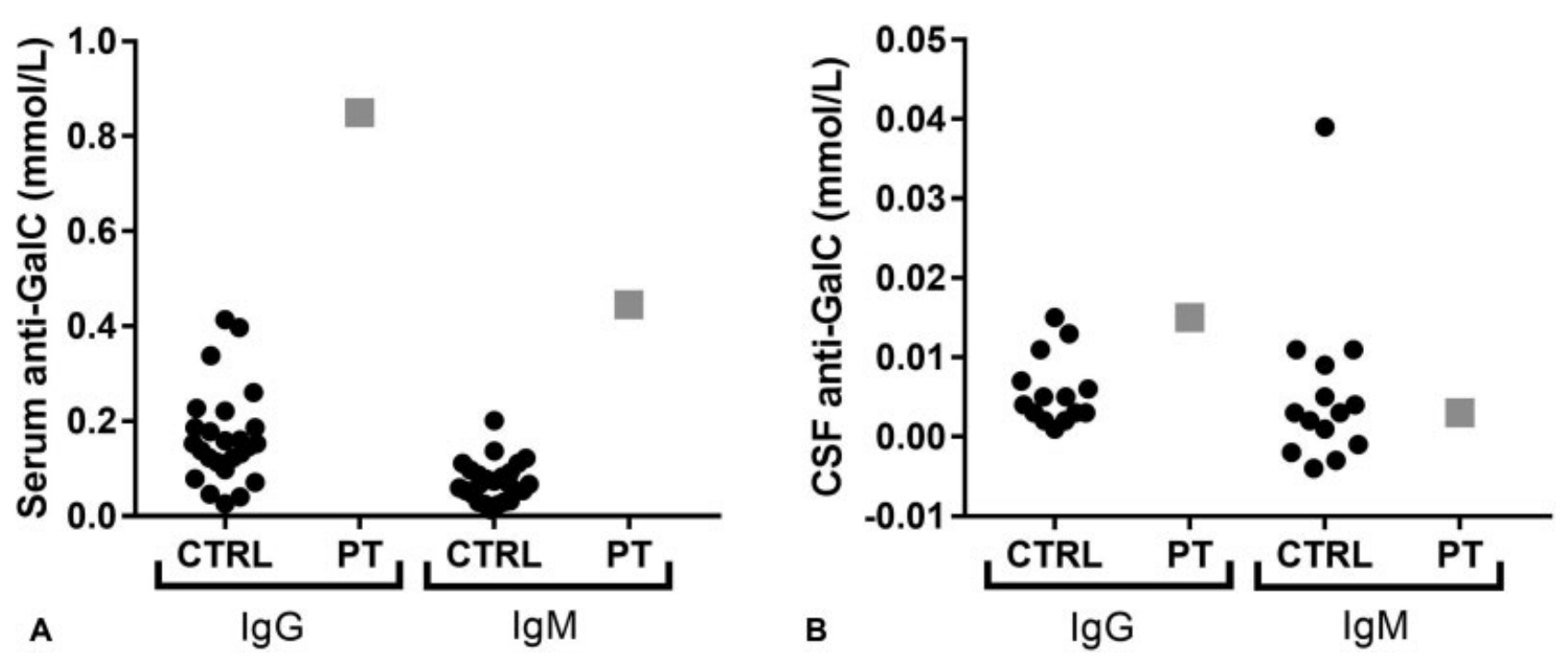

Fig. 2 Quantification of GalC IgG and IgM antibodies in serum (A) and CSF (B) in control samples (black dots) and our patient (grey square). GalC, galactocerebroside. 
Our patient does well at home and school, and levetiracetam was discontinued in the absence of seizures.

M. pneumoniae carriage is prevalent. Genetic material can be encountered in the airways of $21-56 \%$ of children without respiratory symptoms but may vary substantially with season and year. ${ }^{1,2}$ In a case series of children presenting with acute encephalitis, 50/159 cases demonstrated evidence of a (previous) $M$. pneumoniae infection but this was only judged to be probably causally related in $11 / 159$ cases and possibly in 9/159 cases. $^{3}$ Only in 6/159 cases, CSF PCR for M. pneumoniae was positive. Interestingly, edema of the basal ganglia was described as MRI finding in one of the cases. ${ }^{3}$ A M. pneumoniae-associated acute onset bilateral striatal necrosis has previously been described in a girl with neutropenic fever also lacking intrathecal molecular of serological positive tests. ${ }^{4}$ Besides direct infection, M. pneumoniae is associated with postinfectious autoimmune-sequelae in children, including neurological disorders as the Guillain-Barré syndrome (GBS). Recently, in a Dutch cohort of children with GBS, 21\% showed IgM positivity for M. pneumoniae, indicating a recent immune response to the microorganism possibly by infection. ${ }^{5}$ Interestingly, $25 \%$ of children with GBS has elevated serum antibodies to the glycolipid GalC that resides in both the central and peripheral nervous system. ${ }^{5}$ These anti-GalC antibodies show various neurotoxic effects in animal models and may contribute to the development of postinfectious encephalitis or GBS, respectively. $M$. pneumoniae may trigger the production of these anti-GalC antibodies that were shown to cross-react with unidentified structures of the $M$. pneumoniae. ${ }^{6}$ In a Japanese patient series, elevated GalC antibodies were described in patients with an M. pneumoniae-associated encephalitis. ${ }^{7}$ In an earlier case of a 9-year-old boy with Birkenstaf brainstem encephalitis and M. pneumoniae antibodies, elevated GalC IgG and IgM levels were found both in serum and CSF. ${ }^{8}$ Remarkably, GalC antibodies in the CSF of our patient were not elevated. This could be a technical issue with a limited sensitivity of the Enzyme-Linked Immuno Sorbent Assay for detecting GalC antibodies in CSF. The acute and monophasic clinical course of our patient are also compatible with a postinfectious encephalitis following $M$. pneumoniae infection. In previous cases, a short prodrome of 2 days has been argued to be more supportive of an infectious instead of a parainfectious autoimmune etiology. ${ }^{9}$ However, the symptoms of our patient at presentation were likely due to his intracranial disease, a prolonged subclinical prodrome was not recorded. The complete and swift response to treatment with steroids can also be regarded as indication for a parainfectious mechanism.

Our case description supports an association between acute encephalitis with pleiocytosis in children and a M. pneumoniae infect but suggests that besides a primary infect of the CNS, a parainfectious autoimmune phenomenon can be the underlying mechanism. In analogy with GBS, anti-GalC antibodies may be mediators of this postinfectious immune-mediated disease. $^{10}$

\section{Conflict of Interest}

Dr. B.C.J. reports grants from Baxalta, grants from Grifols, grants from CSL-Behring, grants from Annexon, grants from Prinses-Beatrix Spierfonds, grants from GBS-CIDP Foundation International, outside the submitted work. All the other authors report no conflict of interest.

\section{References}

1 Spuesens EBM, Fraaij PLA, Visser EG, et al. Carriage of Mycoplasma pneumoniae in the upper respiratory tract of symptomatic and asymptomatic children: an observational study. PLoS Med 2013; 10(05):e1001444

2 Wood PR, Hill VL, Burks ML, et al. Mycoplasma pneumoniae in children with acute and refractory asthma. Ann Allergy Asthma Immunol 2013;110(05):328-334.e1

3 Bitnun A, Ford-Jones EL, Petric M, et al. Acute childhood encephalitis and Mycoplasma pneumoniae. Clin Infect Dis 2001;32 (12):1674-1684

4 van Buiren M, Uhl M. Images in clinical medicine. Bilateral striatal necrosis associated with Mycoplasma pneumoniae infection. $\mathrm{N}$ Engl J Med 2003;348(08):720

5 Meyer Sauteur PM, Huizinga R, Tio-Gillen AP, et al. Mycoplasma pneumoniae triggering the Guillain-Barré syndrome: a case-control study. Ann Neurol 2016;80(04):566-580

6 Ang CW, Tio-Gillen AP, Groen J, et al. Cross-reactive anti-galactocerebroside antibodies and Mycoplasma pneumoniae infections in Guillain-Barré syndrome. J Neuroimmunol 2002;130(1-2)179-183

7 Nishimura M, Saida T, Kuroki S, et al. Post-infectious encephalitis with anti-galactocerebroside antibody subsequent to Mycoplasma pneumoniae infection. J Neurol Sci 1996;140(1-2)91-95

8 Meyer Sauteur PM, Relly C, Hackenberg A, et al. Mycoplasma pneumoniae intrathecal antibody responses in Bickerstaff brain stem encephalitis. Neuropediatrics 2014;45(01):61-63

9 Narita M, Yamada S. Two distinct patterns of central nervous system complications due to Mycoplasma pneumoniae infection. Clin Infect Dis 2001;33(06):916-917

10 Meyer Sauteur PM, Jacobs BC, Spuesens EBM, et al. Antibody responses to Mycoplasma pneumoniae: role in pathogenesis and diagnosis of encephalitis? PLoS Pathog 2014;10(06):e1003983 